\title{
The Impact of Atypical Early Histories on Pet or Performer Chimpanzees
}

It is widely accepted that an animal's early history, including but not limited to its rearing history, can have a profound impact on later behavior. In the case of captive animals, many studies have used categorical measures such as mother reared or human reared that do not account for both the influence of human and conspecific interaction. In order to account for the influence of both human and conspecific early exposure to later behavior, we collected 1385 hours of data on 61 chimpanzees, of which 36 were former pets or performers, currently housed at accredited zoos or sanctuaries. We developed a unique metric, the ChimpanzeeHuman Interaction $(\mathrm{CHI})$ Index that represented a continuous measure of the proportion of human and chimpanzee exposure subjects experienced and here focused on their exposure during the first four years of life. We found that chimpanzees who experienced less exposure to other chimpanzees as infants showed a lower frequency of grooming and sexual behaviors later in life which can influence social dynamics within groups. We also found chimpanzees who experienced more exposure to other chimpanzees as infants showed a higher frequency of coprophagy, suggesting coprophagy could be a socially-learned behavior. These results help characterize some of the long-term effects borne by chimpanzees maintained as pets and performers and may help inform managers seeking to integrate these types of chimpanzees into larger social groups, as in zoos and sanctuaries. In addition, these results highlight the necessity of taking into account the time-weighted influence of human and conspecific interactions when assessing the impact that humans can have on animals living in captivity. 
2 Hani D. Freeman and Stephen R. Ross

3 Lester E. Fisher Center for the Study and Conservation of Apes, Lincoln Park Zoo, IL, USA

4 Correspondence should be sent to Stephen Ross, sross@1pzoo.org 


\section{Introduction}

6 Early life experiences have a significant impact on the behavioral development or the way

7 in which individuals, both human and non-human, learn to interact with their environment (e.g.

8 humans, Kagan, 1996; Fox \& Henderson, 1999; non-human primates, Parker \& Maestripieri,

9 2011). Outcomes of early life experiences have uncovered a broad range of potential impacts

10 including those falling in both the social and non-social realms. Non-social variables include the

11 impact of the physical environment on development (rats, Leshem \& Schulkin, 2012), as well as

12 physiological factors such as genetics (primates Barr et al., 2003; Suomi, 2011) or hormones

13 (primates, Saltzman \& Maestripieri, 2011) that influence maternal care. Studies of social effects,

14 including both maternal and non-maternal influences, have focused primarily on the impact of

15 conspecifics in an infant's environment and the subsequent effect on behavior expressed later in

16 life (humans, Sroufe, 2005; rodents \& primates, Pryce et al., 2005; primates, Suomi, 1997).

17 Even animals typically considered less social in nature, such as lizards, seem vulnerable to the

18 effects of atypical and impoverished social histories (Cissy, Richard \& Mats, 2014).

A range of circumstances in captivity might require infants to be raised by humans rather

20 than by peers or their mothers. For instance, a biological mother may be unable to care for her

21 offspring due to illness or disinterest leading to neglect; in these circumstances, human

22 intervention may be warranted. Studies investigating the impact of these atypical rearing

23 situations on primates - notably with rhesus macaques and chimpanzees - have used categorical

24 classifications, such as mother-reared and human-reared, and reported a range of substantive

25 impacts on developmental trajectories (Anderson \& Mason, 1974; Maki, Fritz \& England, 1993;

26 Suomi, 2011). In the most extreme cases of social deprivation, there are a range of impacts

27 including behaviors such as self-clutching, excessive rocking, and self-mutilation (Harlow,

28 Dodsworth \& Harlow, 1965). In laboratory settings, peer-reared chimpanzees (with no maternal

29 contact) tended to be less dominant and less active and often showed more abnormal rocking and 
30 less social play behavior (Rosenblum \& Kaufman, 1968; Bloomsmith, Lambeth \& Alford, 1991;

31 Spijkerman et al., 1994; Spijkerman, 1996). Alternatively, a recent study assessing orphaned and

32 mother-reared chimpanzees in African sanctuaries found that orphaned chimpanzees engaged in

33 more social play compared with those classified as mother-reared. However, the play periods of

34 the orphaned chimpanzees were shorter and more often led to aggression (Van Leeuwen,

35 Mulenga, \& Chidester, 2014). The effects of these early rearing histories may have long-term

36 effects as well: studies of laboratory-housed chimpanzees that were raised exclusively by humans

37 exhibited more abnormal behaviors later in life in comparison to peer-reared chimpanzees who

38 were raised with other chimpanzees (Martin, 2002; Martin, 2005). In sum, the results of these

39 studies show human-reared chimpanzees seem particularly prone to both short- and long-term

40 negative impacts on their behavior and likely their wellbeing.

41 Although previous studies have noted both short - and long-term impacts on chimpanzees

42 who were human-reared, past research on human-reared chimpanzees has not taken into account

43 differences in the degree and amount of human interaction in early development and how this

44 influences later behavior. However, social influences may vary depending on an individual's

45 living situation, so a strict categorical classification may not be adequate in many cases. For

46 example, the largely unstudied population of privately-owned chimpanzees - those living as

47 personal pets and as trained performers in the entertainment industry - often includes individuals

48 who have experienced a mix of human and chimpanzee influences. A continuous metric that

49 allows researchers to account for variation in social influences at different stages of an

50 individual's life may be more apt than a categorical metric, so that a wide variety of potential

51 influences can be assessed.

52 Chimpanzees bred for the pet industry are typically removed from their mother soon

53 after birth to facilitate human handling. They are sold to members of the general public, who

54 most often have no experience or training to care for this species. These pet chimpanzees are 
55 likely to have relatively little exposure to conspecifics early in life and during key developmental

56 periods. Many are essentially raised as humans, with related traditions such as eating at a table

57 and wearing clothes, until they grow to be too large and dangerous to be kept in the home.

58 Performing chimpanzees have a more variable trajectory and though they may spend some

59 proportion of their time with other chimpanzees, they are also highly exposed to humans (trainers

60 and audiences) until they too typically grow to be unmanageable in adolescence. The categorical

61 designations of "human-raised", "peer-raised", or "mother-raised" do not encompass the actual

62 experience of a chimpanzee such as those who performed and spent significant time in full

63 contact with both humans and other chimpanzees. There is growing consensus that privately-

64 owned chimpanzees represent significant human health and safety risks (McCann et al., 2007).

65 In the case of entertainment chimpanzees, these practices produce additional consequences such

66 as negative public perceptions that can impact conservation efforts (Ross et al., 2008; Ross,

67 Lonsdorf \& Vreeman, 2011; Schroepfer et al. 2011). However, here we focus on the long-term

68 outcomes of these practices and the degree to which the atypical early histories experienced by

69 these chimpanzees influence their behavioral development.

70 In the current study, we used a novel approach to assess the impact of atypical early

71 histories experienced by pet and performing chimpanzees and the subsequent outcomes for

72 behavioral development. We employed a long-term continuous measure, chimpanzee-human

73 index (CHI), that accounts for both the amount of time spent with humans as well as the amount

74 of time spent with other conspecifics. We examined how differential human/conspecific exposure

75 during the infant period or the first four years of life impacted current behavioral patterns for ex-

76 pet and ex-performer chimpanzees now living in accredited zoos and sanctuaries. Although our

77 question revolved around chimpanzees without early exposure to conspecifics, we also studied

78 the behavioral patterns of chimpanzees who have lived their entire lives in their natal group to

79 provide a comparison group. These zoo-born chimpanzees may also have a range of 
80 human/conspecific exposure early in life; however, most have never had full contact with humans

81 and have always lived with other chimpanzees. In the current study, we focused on the first four

82 years of life because this is considered to be a particularly influential period for behavioral and

83 socio-cognitive development for this species (Tomasello, Kruger \& Ratner, 1993; Bard, 1995;

84 Bard et al., 2014). We predicted that chimpanzees with atypical early histories (high human

85 exposure, low conspecific exposure) would differ behaviorally compared with those having more

86 species-typical histories. We projected these differences would be most pronounced in areas of

87 social, sexual and abnormal behaviors and ultimately reflect our hypothesis that these atypical

88 early histories can result in a long-term decrease in species-typical developmental trajectories .

\section{Materials and Methods}

90 This research was conducted at three sanctuaries that are members of the North American

91 Primate Sanctuary Alliance (NAPSA: Center for Great Apes, Chimps, Inc., and Save the Chimps)

92 and six zoos accredited by the Association of Zoos and Aquariums (AZA: Houston Zoo, Dallas

93 Zoo, Lincoln Park Zoo, Lion Country Safari, North Carolina Zoo, and Oakland Zoo). Each of

94 these facilities adheres to high standards of chimpanzee care including housing chimpanzees in

95 social groups and providing nesting material and various forms of enrichment. All animals were

96 observed in their home cages during observations. All subjects had ad libitum access to water

97 and at no time were the subjects ever food or water deprived. Subjects were provided daily with

98 primate chow and fruit and/or vegetable food enrichment at each of the facilities. This study was

99 approved by and complied with protocols approved by the Chimpanzee Species Survival Plan

100 (SSP) management group as well as animal care committees at each of the institutions that

101 participated in this study.

\section{Subjects}


10454 years) that varied widely in the degree of human and conspecific exposure they experienced 105 early in their lives (Figure 1, Tables S1 in Online Supplementary Materials). Though many of 106 them were formerly housed as personal pets or performers, all were evaluated in their current

107 housing at NAPSA sanctuaries or AZA zoos. The number of subjects studied at each institution 108 ranged from four to 12 . The subjects had been at his/her respective facilities between one month 109 and 54 years before the study. The average amount of time that each subject spent at his/her 110 respective facility before the study was nine and a half years. Data were collected at three

111 NAPSA-accredited sanctuaries as well as six AZA-accredited zoos. None of the NAPSA

112 sanctuaries were open to the public, but the AZA zoos all had public access with daily visitors.

113 All of the subjects were socially housed, with between one and 25 other chimpanzees (average

114 group size was seven chimpanzees). All of the chimpanzees in the study were captive-born, and 115 none had lived in a laboratory environment.

\section{Behavioral Assessments}

117 Behavioral data on the chimpanzees were collected with a modified version of an

118 ethogram used for Lincoln Park Zoo's long-term behavioral monitoring studies of great apes

119 (Ross et al., 2011). The ethogram included six primary behavioral categories (social (e.g.

120 grooming, playing, begging, embrace) sexual, agonism, solitary, inactivity and abnormal), which

121 were comprised of 21 behaviors (provided in the Online Supplementary Materials). Behavioral

122 data were collected using a combination of all occurrence and scan sampling. Data were collected

123 with handheld computers (Pocket Observer 2.0, Noldus Observer, Noldus Information

124 Technology, Wageningen, The Netherlands) in 30-min focal samples with a 30-sec intersample

125 interval for the scan samples. When more than one focal sample was collected on the same

126 individual in one day, there was at least a one hour period between each of the samples collected

127 for that individual. Observations were conducted between 9 am and $5 \mathrm{pm}$ by a single observer 
128 (HF) at eight of the nine study sites. The data collected at the ninth site (Lincoln Park Zoo) were

129 collected by observers who had previously achieved $85 \%$ reliability on the ethogram. HF

130 conducted a post hoc inter-rater reliability assessment with a researcher at the Lincoln Park zoo

131 and was found to have $90 \%$ reliability on the ethogram. An equal number of observations were

132 collected in the morning and afternoon for each chimpanzee. The order of the observations was

133 randomly selected ahead of time. Feeding and enrichment times varied at each of the study sites,

134 and data were collected to cover each of these periods. Observations were conducted from a safe

135 and approved area from where the chimpanzees could be easily seen but would not be unduly

136 affected by the presence of the observer. Between 14.5 and 30 hours of data were recorded on

137 each subject, over a period ranging from three to eight weeks between November 2011 and

138 November 2012, for a total of 1385 hours of behavioral data. The number of hours varied due to

139 time constraints and the number of subjects on which data were collected at each institution. The

140 range in the number of hours collected on each subject was factored into the analyses.

\section{Chimpanzee Human Interaction Index}

142 In order to characterize the variable degree of exposure to potential influences

143 (conspecifics and humans), we developed a novel, continuous measure, the Chimpanzee Human

144 Interaction (CHI) index. We used management records acquired from past and current holding

145 institutions and calculated the proportion of time per day that each chimpanzee spent in each of

146 three categories: full exposure to conspecifics, full exposure to humans, and mixed exposure to

147 both conspecifics and humans. Each day was assigned a numerical value based on these three

148 categories of exposure. For instance, chimpanzees living exclusively within a large social group

149 in a zoo with only minimal exposure to humans would have a proportion of $1 / 1$ for the day,

150 indicating they spent $100 \%$ of their time with other chimpanzees. Likewise, a pet chimpanzee

151 raised exclusively with a human family, without any exposure to other chimpanzees, would have 
152 a proportion of $0 / 1$ for that same day. A performing chimpanzee with relatively equal exposure to

153 small groups of conspecifics and full contact with human trainers and audiences would have a

154 proportion of $0.5 / 1$. CHI is calculated as the sum of these variable exposure periods over a

155 particular timeframe given that many chimpanzees have experienced variation in the degree of

156 human and conspecific exposure across their lifetimes. For this analysis, we chose to focus

157 specifically on the infant period: the first four years of life (see Figure 1 for a histogram of the

158 infant $\mathrm{CHI}_{\mathrm{i}}\left(\mathrm{CHI}_{\mathrm{i}}\right.$ refers to the $\mathrm{CHI}$ value of chimpanzees during the infant period) distribution

159 and Table S1 in Online Supplementary Materials for details about each subject), however the CHI

160 index could be utilized to characterize human/conspecific exposure across any particular

161 timeframe or across the entire lifetime.

\section{Categorical Groups}

163 Although our $\mathrm{CHI}$ index was developed to be used as a continuous variable, $\mathrm{CHI}_{\mathrm{i}}$ values

164 in this study revealed a non-normal distribution of the data with clear peaks (Figure 1). This was

165 likely a result of relatively low variability in conspecific/human exposure over the first four years

166 of life. Therefore, we analyzed the data using categorical groupings by early history experience:

167 with subjects categorized as having only or primarily human exposure $\left(\mathrm{CHI}_{\mathrm{i}}\right.$ index: $0-0.30, \mathrm{n}=$

168 6), exposure to both chimpanzees and humans $\left(\mathrm{CHI}_{\mathrm{i}}\right.$ index: $\left.0.31-0.70, \mathrm{n}=32\right)$, or primarily

169 chimpanzee experience $\left(\mathrm{CHI}_{\mathrm{i}}\right.$ index: .71 - 1.0, $\left.\mathrm{n}=21\right)$ (see Table $\mathrm{S} 1$ in the Online Supplementary

170 Materials for each subject's rating).

\section{Data Analysis}

172 Data analyses were conducted in IBM SPSS 20 (IBM Corp., 2011). A false discovery rate

173 correction was performed on the results to control for multiple comparisons, and a corrected

174 alpha value of $\mathrm{p}$ less than or equal to .01 was considered to be significant for all tests. We

175 controlled for the difference in the number of hours spent observing each chimpanzee by 
176 calculating the proportion of time out of the total that an individual spent engaging in a particular

177 behavior compared to the total number of hours of observations on the individual.

178 In order to assess differences between the three categories of early history during the

179 infant period (“human," "mixed," and "chimpanzee”), we performed a one-way multivariate

180 analysis of variance (MANOVA) using each of the 21 behaviors as dependent variables and the

181 early history categories as a fixed factor.

\section{Results}

One-way MANOVA analysis revealed there was a statistically significant difference in the

184 proportion of time the chimpanzees spent engaging in different behaviors based on their early

185 exposure history $\left(F(44,70)=3.180, p<.00005\right.$, Wilks' Lamda $=.111$, partial $\left.\eta^{2}=.667\right)$.

186 Separate analyses revealed that sex, age, potential number of social partners, and time spent at

187 current location did not significantly change the results $(\mathrm{p}>.05$ for all behaviors after controlling

188 for the effect of early history). A series of one-way ANOVA's on each of the 21 dependent

189 variables was conducted as follow-up tests to the MANOVA. An examination of the data revealed

190 that for the following behaviors, the homogeneity of variance assumption was violated: social

191 sex, masturbation, abnormal movement, coprophagy, abnormal body posturing, and abnormal

192 plucking. In these cases, we ran a Welsh test on the data to look at the significance of the ANOVA

193 test. The results of the Welsh test revealed that for the behaviors of abnormal movement and

194 abnormal plucking, the F-value, which was significant in the original ANOVA test, was not

195 significant after correcting for unequal variances. However, the ANOVA tests for the remaining

196 behaviors revealed that in four (social, sexual, inactivity, abnormal) out of six behavioral

197 categories a significant relationship existed between categories of early history and the proportion

198 of time subjects were observed engaging in each behavior. The results of the individual ANOVA

199 tests between proportion of behaviors and categories can be seen in Tables 1, 2 and 3. In addition, 
200 a series of post-hoc analyses (Dunnett's T3 assuming unequal variances) were performed to

201 examine individual mean difference comparisons across the three levels of $\mathrm{CHI}_{\mathrm{i}}$ values and each

202 of the behaviors found to be significant with the MANOVA at $\mathrm{p}<.05$. The specific results are

203 outlined below.

\section{Social Behavior}

205 The individual ANOVA analyses revealed that early exposure categories influenced frequencies 206 of grooming behavior later in life. The ANOVA revealed a significant difference between groups 207 in frequency of giving grooming $(F(2,56)=10.13, p=0.0001)$. Post-hoc analyses revealed that 208 subjects with high amounts of chimpanzee exposure early in life groomed significantly more than 209 those with mixed $(p=0.012)$ or minimal exposure to conspecifics $(p=0.002)$.

210 There was also a significant difference between groups in frequency of grooming received

$211(F(2,56)=9.22, p=.0001)$. Post-hoc analyses revealed that the subjects with high amounts of

212 chimpanzee exposure early in life groomed significantly more than those with mixed $(\mathrm{p}<$

$2130.0001)$ or minimal exposure to conspecifics $(\mathrm{p}=0.003)$. There was not a significant relationship

214 between early exposure category and frequencies of social play or other prosocial behaviors $(p$ $215>.01)$

\section{Sexual Behavior}

217 The ANOVA revealed a significant difference between groups in frequency of sexual

218 behavior $(F(2,56)=4.98, p=.01)$. The post-hoc analyses revealed that chimpanzees with high

219 amounts of early exposure to conspecifics demonstrated higher frequencies of mounting and

220 sexual exploration compared to chimpanzees with low exposure to conspecifics $(p=.02)$. . There

221 was not a significant difference found between chimpanzees with high exposure to conspecifics 
222 and those with mixed exposure $(\mathrm{p}>.01)$. There was not a significant relationship found between

223 the category of early exposure and rates of masturbation $(p>0.01)$.

\section{Agonistic and Solitary Behaviors}

225 There was no difference in the expression of agonistic or solitary behaviors, including

226 displays, non-contact, contact aggression given or received, self-grooming or self-play in relation 227 to categories of early exposure $(p>.01)$.

\section{Inactivity}

229 The ANOVA analyses revealed significant differences in frequencies of inactivity between 230 the early history categories $(F(2,56)=7.72, p=.001)$. Post-hoc tests revealed that chimpanzees 231 with mixed early exposure demonstrated higher rates of inactivity than those with either primarily 232 human $(p<.0001)$ or primarily conspecific exposure early in life $(p=.002)$.

\section{Abnormal Behavior}

234 We examined four forms of abnormal behavior: coprophagy, abnormal movement,

235 abnormal body posturing, and hair plucking. The ANOVA analysis revealed significant

236 differences in frequencies of coprophagy between the early history categories $(F(2,56)=14.99$,

$237 p=.0001)$. The post-hoc tests revealed that chimpanzees with high amounts of early exposure to 238 conspecifics demonstrated the highest frequencies of coprophagy later in life than those with 239 either mixed early exposure $(p=.040)$ or primarily human exposure early in life $(p=.001)$. There

240 was not a significant difference in rates of coprophagy between chimpanzees from primarily

241 human or mixed early exposure histories. The ANOVA analysis found there was not a significant

242 difference between the three categories in rates of the other forms of abnormal behavior $(p>$.

$24301)$. 


\section{Discussion}

245 The primary aim of this study was to use a novel approach to assess the long-term

246 behavioral impact of variable early life exposure to both conspecifics and humans on captive

247 chimpanzees. To achieve this, we focused on a rarely studied population, former pet and

248 performer chimpanzees that now live in accredited zoos and sanctuaries with other chimpanzees.

249 We also studied individuals who had experienced more typical early life histories for captive

250 chimpanzees, living in their natal group with multiple conspecifics, in accredited zoo

251 environments throughout their lifetime. In general, supportive of our hypotheses, we found that

252 chimpanzees raised in "human" or "mixed" exposure groups tended to exhibit lower frequencies

253 of grooming compared with those in the "chimpanzee" group. In addition, chimpanzees raised in

254 the "human" exposure group exhibited lower frequencies of social sexual behavior later in life as

255 compared with those in the "chimpanzee" group. Lastly, chimpanzees in the "mixed" exposure

256 group exhibited higher frequencies of inactivity compared with the "human" or "chimpanzee"

257 groups. The results of this study suggest that high human exposure early in a chimpanzee's life,

258 and/or reduced conspecific exposure, is related to observable differences in behavior in

259 adulthood. Grooming and sexual behavior are important components to the dynamics of social

260 groups in chimpanzees. Decreases in these behaviors could have the potential to be related to

261 animal management and welfare issues connected with social interactions between chimpanzees.

262 While it is possible that variability in current living environments may represent a

263 confound to the conclusions of this study (due in part to the relatively limited number of

264 institutions caring for chimpanzees with such atypical backgrounds), it is important to note that

265 the demonstrated effects are unlikely to be solely the result of current physical or social

266 environments. All subjects were socially housed, and all were managed under similar

267 contemporary management systems. 
The behavioral differences observed between early-history groups are consistent with

269 previous findings in chimpanzees and other non-human primates (i.e. mother-reared individuals

270 compared to isolated individuals, Turner, Davenport \& Rogers, 1969; Davenport \& Rogers, 1970;

271 Harlow \& Suomi, 1971; Kalcher et al., 2008). For example, several studies have demonstrated

272 that human-reared primates often have lower rates of reproductive success, possibly indicative of

273 less appropriate sexual behavior (Ryan, Thompson \& Roth, 2002). The evidence for a

274 developmental influence of rearing on later chimpanzee behavior is mixed. While some studies

275 report a lack of rearing effect in social behavior (Bloomsmith et al., 2002; Howell et al., 2006;

276 van Ijzendoorn et al., 2009) more recent analyses (Clay, 2012) and the results of our analyses

277 suggest the possibility that these effects might not be evident until much later in life. Duration of

278 exposures of chimpanzees to humans or conspecifics might also be a particularly important

279 factor. Martin (2005) assessed behavior in chimpanzees that were reared in a variety of contexts

280 and found no statistical effect of rearing; however, the authors were unable to account for the

281 duration of time spent in each of the rearing categories.

282 We also noted differential effects of early experiences on coprophagy, a behavior typically

283 categorized as abnormal (Walsh, Bramblett \& Alford, 1982; Nash et al., 1999). We found that

284 chimpanzees with more conspecific exposure engaged in coprophagy more frequently compared

285 to those in the "mixed" or "human" groups. This behavior is likely socially learned (as opposed

286 to an individually-developed response indicative of stress) and thus animals with broad social

287 exposure may be more likely to adopt these behaviors. Previous studies have found that mother-

288 reared chimpanzees engage in coprophagy more often than non-mother-reared individuals (Nash

289 et al., 1999; Bloomsmith et al., 2005), which suggests that coprophagy may be functionally

290 distinct from other abnormal behaviors. Future studies should involve investigating potential

291 welfare concerns associated with different types of abnormal behaviors in order to determine

292 which behaviors are performed only during times of stress compared with other times. Despite 
293 the breadth of the data used for this investigation, there remain a number of limitations that

294 reflect the complexity of a multi-institutional study and require additional consideration. The

295 first is the potential confound between a chimpanzee's current housing locations with his/her

296 early history exposure. Chimpanzees from particular backgrounds tended to cluster at specific

297 institutions. As a result, there was relatively little intra-institutional variation in early histories

298 among the chimpanzees housed within single groups. Fortunately, the variation in current

299 housing conditions and management styles was relatively low, at least in comparison to the wide

300 variety of physical and social environments from which many of these chimpanzees originated.

301 All current housing was either at AZA-accredited zoos or NAPSA member sanctuaries; all

302 maintain consistently high standards of care in terms of diet, enrichment, and housing.

303 Additionally, subjects were housed in different social groupings (ranging from $2-26$

304 chimpanzees per group), and in some cases, this was an outcome of their atypical early histories

305 and difficult socialization histories. In addition, some of the chimpanzees were genetically related

306 to each other; however, because of the amount of variability in the degree of relatedness both

307 within and across institutions, we could not account for it given our current sample size.

308 We must also consider the potential limitations of our measure of human and conspecific

309 exposure, including both the CHI index and categories based on this index. In this analysis we

310 utilized a subset of the $\mathrm{CHI}$ index $\left(\mathrm{CHI}_{\mathrm{i}}\right)$, specific to a relatively narrow time frame from birth to

311 age 4. During this timeframe, there is inherently less variation across individuals in comparison

312 to that observed across the entire lifetime. For instance, chimpanzees with low conspecific

313 exposure were usually maintained in those conditions across those first four years, but across

314 their lifetime they may experience a wider array of social conditions. Subsequently, we chose to

315 use a categorical analysis. Only six of the 60 chimpanzees had solely human exposure during

316 infanthood but including this category in the analyses was important to draw comparisons among

317 those individuals who lacked exposure to conspecifics. Inclusion of this small sample group 
318 comes with limitations including the possibility of failing to detect some behavioral effects (type

319 II errors). We also acknowledge that it is possible that individuals with similar CHI values may

320 in fact have experienced very different patterns of exposure to conspecifics and humans. Further

321 refinement of this index may help address these potential limitations.

322 Overall, the results of this study suggest that adolescent and adult chimpanzee behavior is

323 associated with early life experiences and that individuals exposed to more human-centric

324 environments may express behavioral deficits later in life in relation to grooming and sexual

325 behavior. It is possible that the consequences of these developmental trajectories may differ

326 considerably for chimpanzees living in situations with greater regular exposure to humans, such

327 as in laboratory settings. Research on how the $\mathrm{CHI}$ index relates to differences in personality,

328 cognitive performance, stress levels (as measured through cortisol), and health histories is likely

329 to give a more comprehensive representation of how these effects are manifested. Finally, care

330 should be taken to consider the substantive variation in how these early histories affected

331 individuals. Not every chimpanzee who had a low $\mathrm{CHI}_{\mathrm{i}}$ score showed deficits in social and

332 sexual behaviors, as evidenced by the wide-ranging standard deviations. Future studies should

333 help identify what variables lead to better social resilience in order to aid chimpanzees who

334 struggle more with social integration. The results of this research suggest that future research

335 should focus on developing the best management strategies for how to care for chimpanzees with

336 a variety of early histories in order to meet their social needs. The reduced (or absent) exposure to

337 conspecifics and full contact exposure to humans that these chimpanzees experienced, especially

338 during the first four years, may have especially profound and long-term behavioral outcomes.

339 Given the known public safety concerns surrounding pet chimpanzee ownership and the negative

340 perception and conservation impacts of inappropriate media portrayals of privately-owned

341 “actor” chimpanzees (McCann et al., 2007; Ross et al., 2008; Ross, Lonsdorf \& Vreeman, 2011), 
342 we now add empirical evidence of the potentially negative welfare effects on the chimpanzees

343 themselves as important considerations in the discussion of privately-owned chimpanzees. We

344 promote further use of these and other evidence-based methods to further inform policy and

345 legislative change that protects chimpanzees and other important non-human animals that are

346 subject to conservation and welfare threats.

\section{Acknowledgements}

We are grateful to Lydia Hopper and Joe Simonis for their help in refining the

349 Chimpanzee/Human Interaction index. The manuscript was greatly improved thanks to the 350 thoughtful feedback provided by Lydia Hopper, the journal editors and four reviewers. We are 351 grateful to Kathy Wagner for her help with using the Observer software for behavioral data 352 collection and to Andrew Steets for his computer programming skills. Thank you to interns at the 353 Lincoln Park Zoo who helped to collect the behavioral data at that facility. We would also like to 354 thank the staff at the Houston Zoo, Dallas Zoo, Lion Country Safari, Center for Great Apes, Save 355 the Chimps, North Carolina Zoo, Chimps Inc. Lincoln Park Zoo, and Oakland Zoo for their 356 support and allowing us to collect data at their facilities. This study was fully funded by a grant 357 through the Arcus Foundation (1102-34).

\section{Author Contributions}

359 This study was designed by SRR in collaboration with HDF. HDF collected the behavioral data 360 at each of the facilities except for the Lincoln Park Zoo. HDF analyzed the data. Both authors 361 contributed to the writing and editing of this article. 


\section{Literature Cited}

363 Anderson CO, Mason WA. 1974. Early experience and complexity of social organization in 364 groups of young rhesus monkeys (Macaca mulatta). Journal of Comparative Physiology 87: 681365690.

366 Barr CS, Newman TK, Becker ML, Parker CC, Champoux, M, Lesch KP, Goldman D, Suomi

367 SJ, Higley JD. 2003. The utility of the non-human primate model for studying gene by

368 environment interactions in behavioral research. Genes, Brain, and Behavior 2: 336-340.

369 Bard KA. 1995. Parenting in primates. In: Bornstein MH, ed. Handbook of parenting.

370 Volume 2, Biology and ecology of parenting. Mahwah: L Erlbaum Associates, 27-58.

371 Bard KA, Dunbar S, Maguire-Herring V, Veira Y, Hayes KG, McDonald K. 2014. Gestures and

372 Social-emotional communicative development in chimpanzee infants. American Journal of

373 Primatology 76: 14-29.

374 Bloomsmith MA, Baker KC, Ross SR, Lambeth SP. 2005. Early rearing conditions and

375 captive chimpanzee behavior: Some surprising findings. In: Sackett GP, Ruppenthal GC, Elias K,

376 ed. Nursery Rearing of Nonhuman Primates in the 21st Century. New York: Kluver Academic

377 Publishers, 289-312.

378 Bloomsmith M, Baker KC, Ross SR, Pazol KA. 2002. The behavioral effects of early rearing

379 experiences on captive chimpanzee behavioral development: The juvenile years. American 380 Journal of Primatology 57(S1):54-55. 
381 Bloomsmith MA, Lambeth SP, Alford PL. 1991. The relationship between social behavior and

382 captive female chimpanzees: Implications for managing chimpanzee (Pan troglodytes)

383 groups. International Journal of Comparative Psychology 4:171-184.

384 Cissy B, Richard S, Mats O. 2014. Effects of early social isolation on the behaviour and 385 performance of juvenile lizards, Chamaeleo calyptratus, Animal Behavior 88:1-6.

386 Clay AW. 2012. Attachment and early rearing: longitudinal effects in chimpanzees (Pan 387 Troglodytes), Ph.D. Dissertation, Georgia Tech University.

388 Davenport RK, Rogers CM. 1970. Differential rearing of the chimpanzee. A project survey. 389 In: Bourne GH, ed. The Chimpanzee, Vol. 3, 337-360.

390 Fox NA, Henderson HA. 1999. Does infancy matter? Predicting social behavior from infant 391 temperament. Infant Behavior and Development 22: 445-455.

392 Harlow HF, Dodsworth RO, Harlow MK. 1965. Total social isolation in monkeys. Proceedings of 393 the National Academy of Sciences 54: 90-97.

394 Harlow H, Suomi SJ. 1971. Social Recovery by Isolation-Reared Monkeys. Proceedings of the 395 National Academy of Sciences 7:1534-1538.

396 Howell S, Schwandt M, Fritz J, Marke M, Murphy J, Young D. 2006. Effects of early rearing 
397 history on growth and behavioral development in captive chimpanzees (Pan troglodytes). In:

398 Sackett GP, Ruppenthal GC, Elias K, ed. Nursery Rearing of Nonhuman Primates in the 21st

399 Century. New York: Kluver Academic Publishers, 313-350.

400 IBM Corp. Released 2011. IBM SPSS Statistics for Windows, Version 20.0. Armonk, NY: IBM 401 Corp.

402 Kagan J. 1996. Three pleasing ideas. American Psychologist 64: 186-196.

403 Kalcher E, Franz C, Crailsheim K, Preuschoft S. 2008. Differential onset of infantile

404 deprivation produces distinctive long-term effects in adult ex-laboratory chimpanzees (Pan 405 troglodytes). Developmental Psychobiology 50: 777-788.

406 Leshem M, Schulkin J. 2012. Transgenerational effects of infantile adversity and enrichment

407 in male and female rats. Developmental Psychobiology. 54:169-86.

408 Maki S, Fritz J, England N. 1993. An assessment of early differential rearing conditions on later 409 behavioral development in captive chimpanzees. Infant Behavior and Development 16:373-381.

410 Martin JE. 2002. Early life experiences: Activity levels and abnormal behaviours in resocialised

411 chimpanzees. Animal Welfare 11:419-436.

412 Martin JE. 2005. The effects of rearing conditions on grooming and play behavior in captive 413 chimpanzees. Animal Welfare 145:125-133. 
414 McCann C, Buchanan-Smith H, Jones-Engel L, Farmer K, Prescott M, Fitch-Snyder H, Taylor S.

415 2007. IPS International Guidelines for the Acquisition Care and Breeding of Nonhuman Primates.

416 International Primatological Society. Available at http://www.internationalprimatologicalso

417 ciety.org/publications.cfm. (accessed 28 April 2011).

418 Nash LT, Fritz J, Alford PA, Brent L. 1999. Variables influencing the origins of diverse

419 abnormal behaviors in a large sample of captive chimpanzees (Pan troglodytes). American

420 Journal of Primatology 48:15-29.

421 Parker KJ, Maestripieri D. 2011. Identifying key features of early stressful experiences that

422 produce stress vulnerability and resilience in primates. Neuroscience and Biobehavioral Reviews

423 R 35: 1466-1483.

424 Pocket Observer 2.0, Noldus Observer, Noldus Information Technology, Wageningen, The 425 Netherlands.

426 Pryce CR, Rüedi-Bettschen D, Dettling AC, Weston A, Russig H, Ferger B, Feldon J.

427 2005. Long-term effects of early-life environmental manipulations in rodents and

428 primates: Potential animal models in depression research. Neuroscience and

429 Biobehavioral Reviews 29 :649-74.

430 Rosenblum LA, Kaufman IC. 1968. Variations in infant development and response to maternal

431 loss in monkeys. American Journal of Orthopsychiatry 38: 418-426. 
432 Ross SR, Lukas KE, Lonsdorf EV, Stoinski TS, Hare B, Shumaker R, Goodall J. 2008.

433 Inappropriate use and portrayal of chimpanzees. Science 319:1487.

434 Ross SR, Lonsdorf EV, Vreeman V. 2011. Specific image characteristics influence attitudes

435 about chimpanzee conservation and use as pets. PLoS ONE 6: e22050.

436 Ross SR., Wagner KE, Schapiro SJ, Hau J, Lukas KE. 2011. Transfer and

437 acclimatization effects on the behavior of two species of African great ape moved to a novel and

438 naturalistic zoo environment. International Journal of Primatology 32: 99-117.

439 Ryan S, Thompson SD, Roth AM. 2002. Effects of hand-rearing on the reproductive success of

440 Western lowland gorillas in North America. Zoo Biology 21: 389-401.

441 Saltzman W, Maestripieri D. 2011. The neuroendocrinology of primate maternal behavior.

442 Progress in Neuro-Psychopharmacology 35: 1192-1204.

443 Schroepfer K, Rosati A, Chartrand T, Hare B. 2011. Use of "entertainment" chimpanzees

444 distorts public perception regarding their conservation status. PLoS One. 6: 26048.

445 Spijkerman R. 1996. Behavioural development of infant chimpanzees with the mother and in 446 peer groups. Annual Report of the Primate Center TNO 1987:309-312.

447 Spijkerman RP, Dienske H, van Hooff JARAM, Jens W. 1994. Causes of body rocking in 448 chimpanzees (Pan troglodytes). Animal Welfare 3:193-211. 
449 Sroufe LA. 2005. Attachment and development: A prospective, longitudinal study from

450 birth to adulthood. Attachment and Human Development 7: 349-367.

451 Suomi S. 2011. Risk, Resilience, and Gene-Environment Interplay in Primates. Journal of 452 the Canadian Academy of Child and Adolescent Psychiatry 204: 289-297.

453 Suomi SJ. 1997. Early determinants of behaviour: Evidence from primate studies. British 454 Medical Bulletin 53: 170-184.

455 Tomasello M. Kruger A, Ratner H. 1993. Cultural learning. Behavioral and Brain Sciences $45616: 495-552$.

457 Turner C H, Davenport RK, Rogers CM. 1969. The effects of early deprivation on the social 458 behavior of adolescent chimpanzees. American Journal of Psychiatry 125: 85-90.

459 Van Ijzendoorn MH, Bard KA, Bakermans-Kranenburg MJ, Ivan K. 2009. Enhancement of 460 attachment and cognitive development of young nursery-reared chimpanzees in responsive versus 461 standard care. Developmental Psychobiology 51: 173-185.

462 Van Leeuwen, E J C, Chitalu Mulenga, I, Lisensky Chidester, D. 2014. Early social deprivation 463 negatively affects social skill acquisition in chimpanzees (Pan troglodytes). Animal Cognition 464 17: 407-414.

465 Walsh S, Bramblett CA, Alford PL. 1982. A vocabulary of abnormal behaviors in restrictively 466 reared chimpanzees. American Journal of Primatology 3:315-319. 


\section{Table 1 (on next page)}

Significant ANOVA Tests with Posthoc Paired Comparisons for Behaviors between Early History Categories During the Infant Period 


\begin{tabular}{|c|c|c|c|c|c|c|}
\hline Behavior & $\begin{array}{l}\text { Early } \\
\text { History } \\
\text { Category }\end{array}$ & $\begin{array}{l}\text { Mean } \\
\text { Percentage } \\
\text { of Time } \\
\text { Engaged in } \\
\text { Behavior }\end{array}$ & SD & $\begin{array}{l}\text { Early } \\
\text { History } \\
\text { Differences }\end{array}$ & $\begin{array}{l}\text { F- } \\
\text { value }\end{array}$ & $\begin{array}{l}\text { Dunnett's T3 } \\
\text { Pairwise } \\
\text { comparisons }\end{array}$ \\
\hline \multirow[t]{3}{*}{ Groom give } & Human & 2.40 & 3.27 & H-M & 10.132 & .930 \\
\hline & Mixed & 3.20 & 3.65 & $\mathrm{H}-\mathrm{C}$ & & $.002 *$ \\
\hline & Chimpanzee & 8.61 & 5.73 & $\mathrm{M}-\mathrm{C}$ & & $.012 *$ \\
\hline \multirow[t]{3}{*}{ Groom } & Human & 1.5 & 1.54 & H-M & 9.219 & .227 \\
\hline & Mixed & 3.1 & 3.20 & $\mathrm{H}-\mathrm{C}$ & & $.000 *$ \\
\hline & Chimpanzee & 6.5 & 3.70 & $\mathrm{M}-\mathrm{C}$ & & $.003 *$ \\
\hline \multirow[t]{3}{*}{ Social sex } & Human & .00 & .00 & H-M & 4.983 & .054 \\
\hline & Mixed & .14 & .22 & $\mathrm{H}-\mathrm{C}$ & & $.020 *$ \\
\hline & Chimpanzee & .54 & .76 & M-C & & .084 \\
\hline \multirow[t]{3}{*}{ Inactive } & Human & 20.30 & 4.06 & H-M & 7.716 & $<.001^{*}$ \\
\hline & Mixed & 33.22 & 12.20 & $\mathrm{H}-\mathrm{C}$ & & .886 \\
\hline & Chimpanzee & 22.10 & 10.20 & M-C & & $.002 *$ \\
\hline \multirow{3}{*}{$\begin{array}{l}\text { Abnormal } \\
\text { Coprophagy }\end{array}$} & Human & .12 & .30 & H-M & 14.994 & .888 \\
\hline & Mixed & .04 & .12 & $\mathrm{H}-\mathrm{C}$ & & $.040 *$ \\
\hline & Chimpanzee & .60 & .58 & M-C & & $.001 *$ \\
\hline
\end{tabular}




\section{Table 2 (on next page)}

Non-Significant ANOVA Tests for Agonism and Abnormal Behaviors between Early History Categories During the Infant Period 


\begin{tabular}{|c|c|c|c|c|c|}
\hline \multicolumn{2}{|r|}{ Behavior } & $\begin{array}{l}\text { Early History } \\
\text { Category }\end{array}$ & $\begin{array}{l}\text { Mean } \\
\text { Percentage } \\
\text { of Time } \\
\text { Engaged in } \\
\text { Behavior }\end{array}$ & SD & F-value \\
\hline \multirow{3}{*}{ 氜 } & \multirow[t]{3}{*}{ Display } & Human & .33 & .50 & \multirow[t]{3}{*}{1.945} \\
\hline & & Mixed & .23 & .23 & \\
\hline & & Chimpanzee & .13 & .19 & \\
\hline \multirow{3}{*}{\multicolumn{2}{|c|}{ NC Aggression receive }} & Human & .00 & .00 & \multirow[t]{3}{*}{.097} \\
\hline & & Mixed & .00 & .00 & \\
\hline & & Chimpanzee & .00 & .00 & \\
\hline & \multirow[t]{3}{*}{ C Aggression receive } & Human & .00 & .00 & \multirow[t]{3}{*}{.270} \\
\hline & & Mixed & .00 & .00 & \\
\hline & & Chimpanzee & .00 & .00 & \\
\hline & \multirow[t]{3}{*}{ NC Aggression give } & Human & .00 & .00 & \multirow[t]{3}{*}{.064} \\
\hline & & Mixed & .00 & .00 & \\
\hline & & Chimpanzee & .00 & .00 & \\
\hline & \multirow[t]{2}{*}{ C Aggression give } & Human & .00 & .00 & \multirow[t]{2}{*}{1.157} \\
\hline & & Mixed & .00 & .00 & \\
\hline \multirow{4}{*}{\multicolumn{2}{|c|}{ Abnormal Movement }} & Human & 6.08 & 8.60 & \multirow[t]{3}{*}{3.492} \\
\hline & & Mixed & 1.63 & 4.22 & \\
\hline & & Chimpanzee & .79 & 2.50 & \\
\hline & & Human & .02 & .03 & \multirow[t]{3}{*}{.746} \\
\hline \multirow{2}{*}{\multicolumn{2}{|c|}{ Abnormal Body }} & Mixed & .05 & .19 & \\
\hline & & Chimpanzee & .00 & .02 & \\
\hline & \multirow[t]{3}{*}{ Abnormal Pluck } & Human & 2.10 & 3.45 & \multirow[t]{3}{*}{6.471} \\
\hline & & Mixed & .27 & .45 & \\
\hline & & Chimpanzee & .44 & .80 & \\
\hline
\end{tabular}




\section{Table 3 (on next page)}

Non-Significant ANOVA results for Solitary, Social and Sexual Behaviors between Early History Categories During the Infant Period 


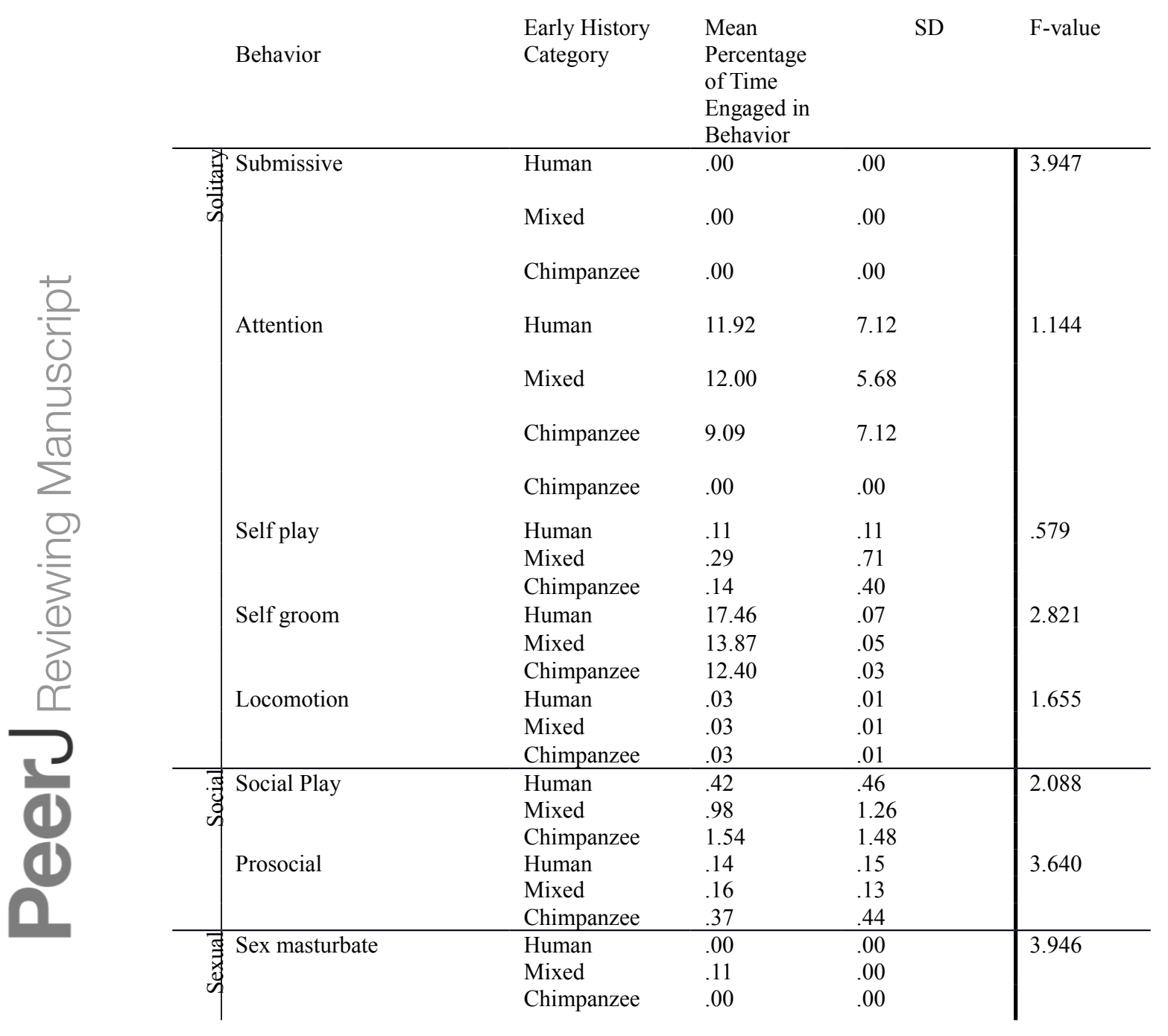




\section{Figure 1}

\section{Histogram of Infant CHI Values}

Distribution of $\mathrm{CHI}_{\mathrm{i}}$ values across chimpanzees in the study.

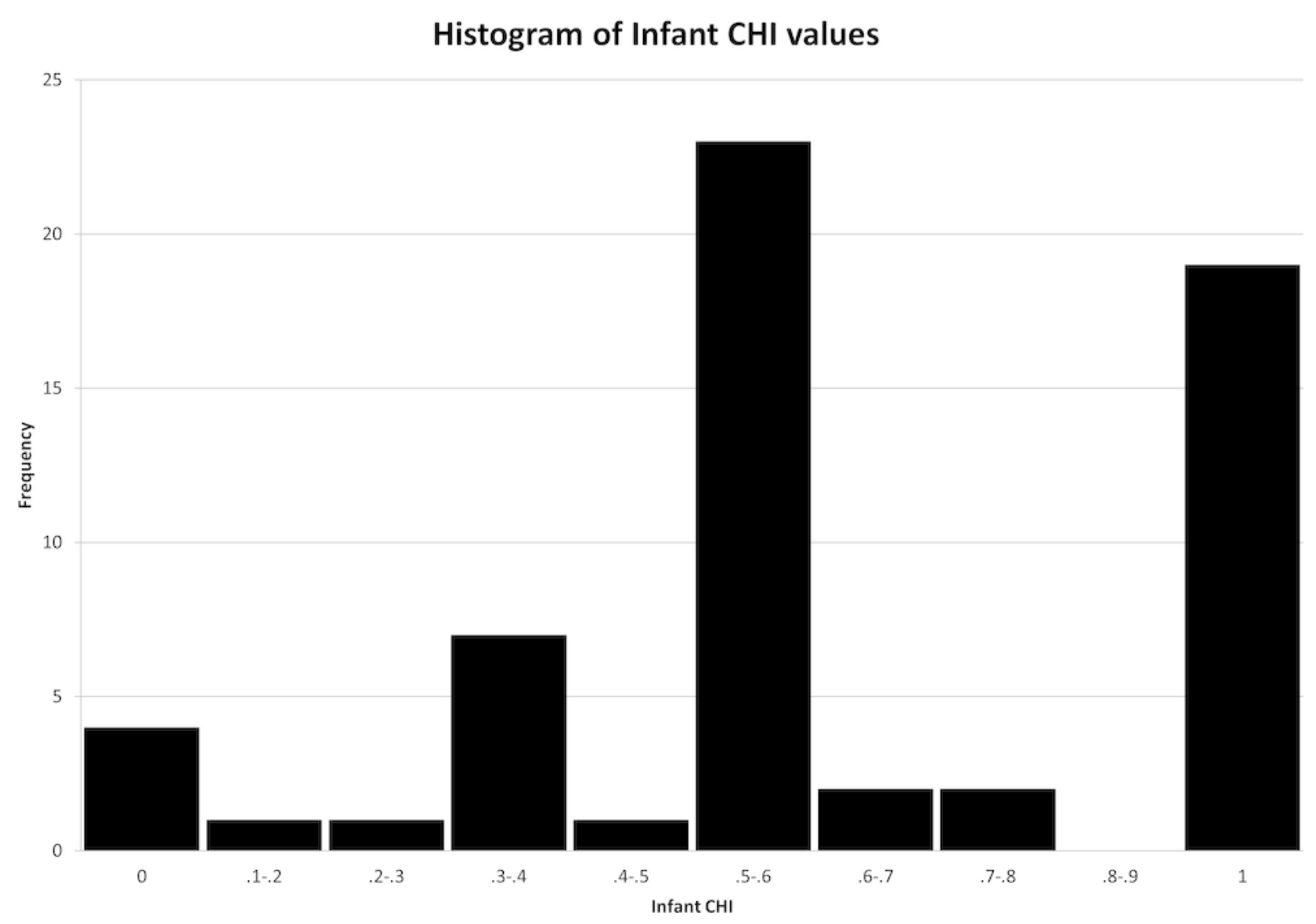

